\title{
Stasis Ulcers - The Unifying Concept
}

\section{Jochanan E. Naschitz}

Bait Balev Nesher and The Ruth and Bruce Rappaport Faculty of Medicine, Technion, Israel Institute of Technology, Haifa, Israel.

Corresponding Author: Jochanan Naschitz, POB 252, Nesher 36602, Derech Hashalom 11. Telephone 073-2-377-300, Fax 97248345575.

Received date: October 25, 2021; Accepted date: December 09, 2021; Published date: December 20, 2021

Citation: Jochanan E. Naschitz. (2021). Stasis Ulcers - The Unifying Concept. J Clinical Research and Reports, 9(4); DOI:10.31579/2690-1919/211

Copyright: (C) 2021, Jochanan E. Naschitz. This is an open access article distributed under the Creative Commons Attribution License, which permits unrestricted use, distribution, and reproduction in any medium, provided the original work is properly cited.

\begin{abstract}
Chronic stasis dermatitis, usually confined to the lower legs, is a complication of longstanding interstitial edema and inflammation, due either to venous hypertension or disorders having in common excessive lymph overload. Heart failure, renal failure, liver cirrhosis, secondary and primary diseases of lymph vessels may complicate with stasis dermatitis. The same mechanisms causing stasis dermatitis can also generate skin ulcers superimposed on stasis dermatitis. In the appropriate context such skin ulcers are called "venous ulcers" or, in different situations, "stasis ulcers". The distinction between venous and other stasis ulcers is usually possible at the bedside. Also, some general measures of therapy are similar for venous and other stasis ulcers: such are elastic compression, topical skin care and ulcer care. In having in common the pathophysiological mechanisms, in bearing clinical resemblance, and responding to similar therapies, a unifying concept may be opportune to comprise the spectrum of stasis dermatitis, venous and other stasis ulcers. The present work is an appeal to this aim.
\end{abstract}

Keywords: stasis dermatitis; calf ulcers; venous hypertension; venous ulcer; lymphedema

\section{Introduction}

According to the traditional concept, as propagated in textbooks of vascular surgery, angiology and dermatology [1-3], stasis dermatitis is a complication of chronic venous insufficiency and is unique to this disease. Venous hypertension is caused by reflux through incompetent valves, venous outflow obstruction, and failure of the calf-muscle pump owing to obesity or leg immobility. Low shear stress, turbulent flow, and especially reversal of flow promote an inflammatory and thrombotic phenotype. In skin-biopsy specimens from lower limbs affected by chronic venous disease there are elevated numbers of macrophages, $\mathrm{T}$ lymphocytes and mast cells. There is increased expression and activity of matrix metalloproteinases (especially MMP-2). Unrestrained MMP activity may contribute to the breakdown of the extracellular matrix, promoting the development of ulcers and impaired healing. Extravasation of red cells leads to elevated ferritin and ferric iron in affected skin. The latter may cause oxidative stress, MMP activation, and a microenvironment that worsens tissue damage [4-7]. The term "chronic venous insufficiency" is generally reserved for venous hypertension of greater severity; varicose veins in the absence of skin changes are not indicative of chronic venous insufficiency [4]. Other skin changes associated with chronic venous hypertension are skin hyperpigmentation, edema, eczema, atrophie blanche (white scar tissue), skin ulcers ("venous ulcers") all localized wih predilection close to the ankel, and lipodermatosclerosis of the lower calf (induration caused by fibrosis of the subcutaneous fat) [8].

Stasis dermatitis is a heterogeneous group of pruritic inflammatory disorders, complicating chronic venous insufficiency but is not unnique to chronic venous insufficiency. In tissues, capillaries and lymphatics are intimately associated and function in coordination to control tissue fluid status. Accumulation of tissue fluid implies insufficiency of the lymphatic system to clear the excess extracellular fluid, due either to lymph overload by excessive blood filtration (high output failure) or, less commonly, by interruption in lymph transport (low output failure). Small and large molecules, including plasma protein, trafficking cells, particulates, and respiratory gases, cross the capillary endothelial barrier, percolate through the tissues, enter the lymph stream, and return to the central venous system. Chronic edema is characterized by trapping the fluid, extravasated proteins and other macromolecules in the skin and the subcutaneous tissues. In the edematous segment there is impaired trafficking of immune cells, abnormal transport of autologous and foreign antigens, mononuclear cell infiltrates (chronic inflammation), epidermal cell and fibroblast proliferation, collagen deposition, altered immunoreactivity, and increased production of local cytokines and growth factors, and progressive obliteration of lymphatics. Whenever tissue fluid stagnates there is vulnerability superimposed cellulitis $[9,10]$. Indeed, as stated by Casley-Smith JR, and recently accepted in consensus, any edema is lymphedema $[11,12]$.

Chronic stasis dermatitis is a persistent, poorly demarcated, scaly, and itchy rash occurring on the lower legs usually in patients with venous disease or in conjunction lymph stasis and edema due to other disorders: heart failure, renal failure, liver cirrhosis, adverse effect of medications [12-16], in primary or secondary diseases of lymph vessels. Researchers suggested that stasis dermatitis represents an allergic response to an epidermal antigen generated through increased hydrostatic pressure. 
Others gave emphasis to an increased susceptibility of the skin to irritation and trauma. Topical medications which contain potential sensitizers such as lanolin, propylene glycol, benzocaine, parabens, calamine, and neomycin could be involved. Allergy to corticosteroids in topical medications is also possible. Stasis dermatitis may be observed in patients with chronic leg edema under the influence of vasodilator drugs such as amlodipine [14]. Recently, we described a first-time stasis dermatitis and calf ulcers occurring under alpesilib treatment; no alternative cause was operative explaining the reaction [15]. A disorder of many etiologies, stasis dermatitis has been labeled a "wastebasket diagnosis" [13].

Chronic stasis dermatitis is easily recognized and clearly distinct from erysipelas. In contradistinction, acute stasis dermatitis appears resembles erysipelas as a red, superficial, itchy plaque emerging rapidly on leg. The diagnosis may be even more challenging when acute stasis dermatitis is the first manifestation of occult venous insufficiency [8].

\section{Discussion}

\section{Stasis calf ulcers}

The effects of the gravitation on the microcirculation produces "homing" of systemic influences to the lower legs. So, the clinician is required to distinguish stasis calf ulcers, venous or otherwise, from calf and foot ulcers of a variety of other causes. Their nosologic diagnosis may be challenging $[17,18]$. Ischemic skin ulcers may be caused by vasculitis, thrombangiitis obliterans, microscopical variant polyangiitis nodosa, cryoglobuninemia, rheumatoid arthritis, Wegener's granulomatosis, systemic lupus erythematosus, nodular vasculitis, cholesterol embolism, antiphospholipid antibody syndrome, calciphylaxis. Hematological disorders or their treatment may cause skin ulcers as reported in sickle cell anemia, warfarin-induced skin necrosis, heparin-induced thrombocytopenia, disseminated intravascular coagulation, essential thrombocythemia, therapy with hydroxyurea. Physical trauma by burns, cold, stings, bites, skin aging may cause skin ulcers. Among metabolic disorders, diabetic skin ulcers are frequent while necrobiosis lipoidica diabeticorum and gouty ulcers are rare. Panniculitides may ulcerate the skin, such Weber Christian disease, pancreatic fat necrosis and alpha 1antitripsin deficiency. The neutrophilic dermatoses pyoderma gangrenosum and Sweet's syndrome cause skin ulcers with certain distinctive features. Infection-induced skin ulcers include ecthyma gangrenosum, furuncle, mycobacteria, Burundi ulcer (caused by Mycobacterium ulcerans), erythema induratum Bazin (caused by Mycobacterium tuberculosis), septic emboli, cutaneous leishmaniasis, cutaneous antrax, syphilis, leprosy, fungal infection, trichophytin granuloma, Cryptococcus neofomans, Coccidioides immitis. Neoplastic skin ulcers include cutaneous T cell lymphoma, basal cell carcinoma, skin metastases, Kaposi sarcoma, squamous cell carcinoma, Merkel cell tumor, malignant transformation of a benign ulcer or scar (Marjolin's ulcer). Because skin ulcers are inherently tissue-destructive and may reflect an underlying systemic disease, there is urgency to arrive at the diagnosis. Yet, stasis ulcers, either venous or other, are usually easily distinguished from other etiologies, except when two or more pathologies co-exist. Lower extremity wounds occurring on the background of chronic edema are generally complex and prone to infection [12].

\section{Different appearance of venous ulcers compared to other stasis ulcers}

Stasis dermatitis and stasis ulcers, either of venous or other origin, have many common traits. However, the "fingerprint" of the primary disorder may be distinctive and dominant (Table 1). So, venous ulcers usually occur in the context of varicous veins and venectasia, have typically perimaleolar location, and usually not much calf edema (Figure 1), rather retractile lipodermatosclerosis may be noticeable. Elephantiasis nostras verucosa (Figure 2) is a rare form of chronic lymphedema, characterized by nonpitting edema and superimposed hyperkeratotic papulo-nodules with a verrucose or cobblestone-like appearance [19]. Leg edema of congestive heart failure, with mild dermatitis and numerous calf ulcers (Figure 3) occurred in the context of anasarca, pleural effusion, massive pitting edema, had now improved on high dose diuretic treatment. The role of recurrent cellulitis in causation of stasis dermatitis and calf ulcer is illustrated by erythema nodosum migrans (Figure 4). In this patient the disorder presented with cutaneous and deep subcutaneous inflammation, and migratory extensive skin ulcers unresponsive to antibiotics as well as corticosteroid treatment. Untreated, erythema nodosum migrans can last for months or years. However, treatment with potassium iodide is usually effective, resulting in clearing of lesions within several weeks [20].

\begin{tabular}{|l|l|l|}
\hline & \multicolumn{2}{|c|}{ Calf ulcers } \\
\hline & "Venous" ulcer & Other stasis ulcer \\
\hline Venous hypertension & +++ & - \\
\hline Varicous veins & +++ & $-/+$ \\
\hline Calf edema & + - & +++ \\
\hline History of calf cellulitis & $+/-$ & + - \\
\hline Lipodermatosclerosis & $+/-$ & - \\
\hline Elephantiasis nostras verucosa & - & + \\
\hline Ulcer perimaleolar & +++ & + \\
\hline Mid and proximal calf ulcer & $+/-$ & +++ \\
\hline Multiple calf ulcers & $+/-$ & ++ \\
\hline Systemic disorder* & - & + \\
\hline
\end{tabular}

Table 1: Severe heart failure, liver cirrhosis, nephrotic syndrome, anasarca, systemic vasculitis, adverse drug reaction (toxic/immune mediated) 


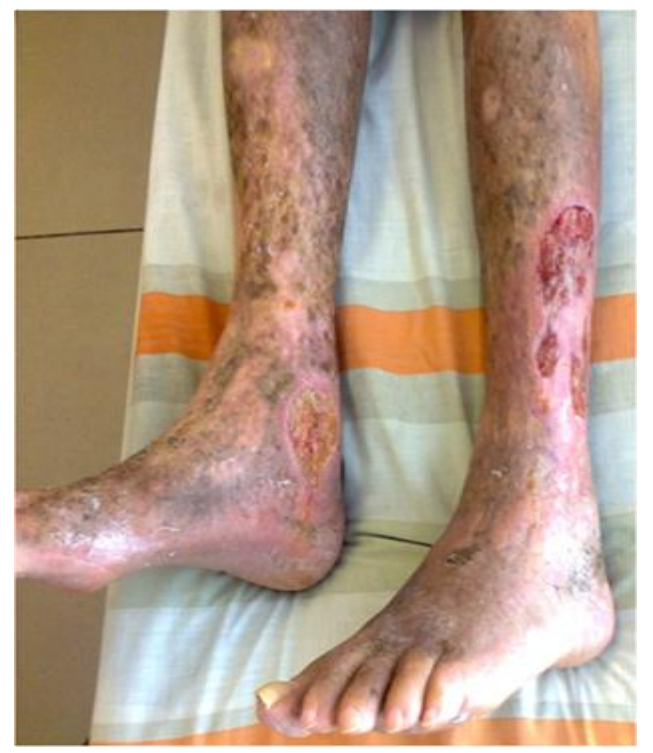

Figure 1: Chronic venous hypertension, perimaleolar and calf ulcers, chronic dermatitis, no leg edema
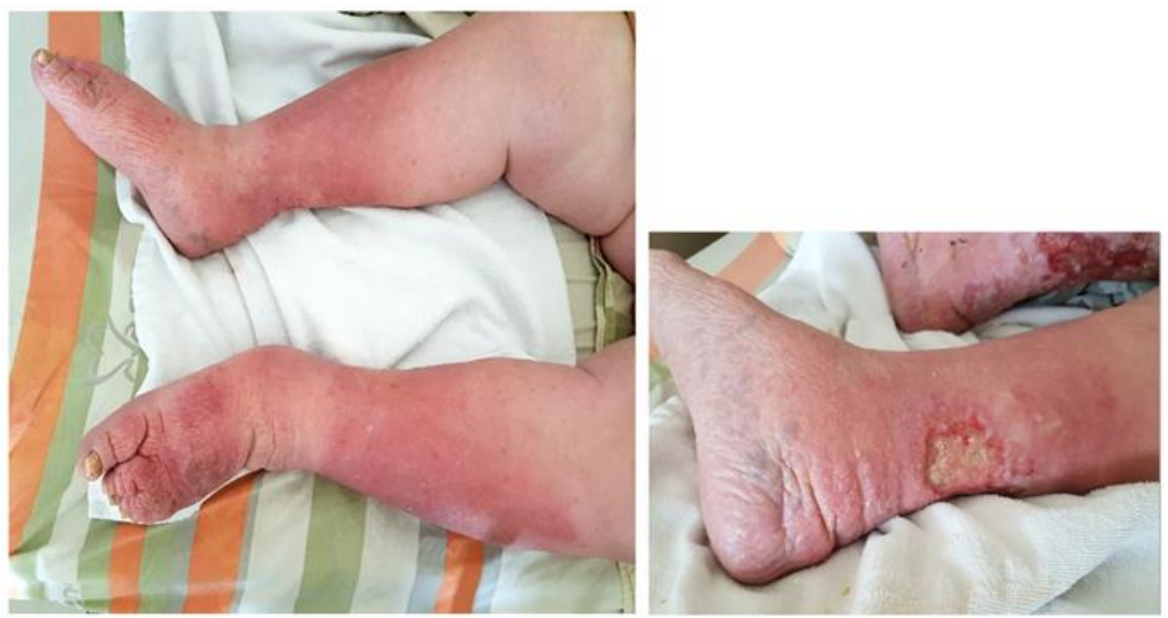

Figure 2: Elephantiasis nostras verucosa - idiopathic lymphedema

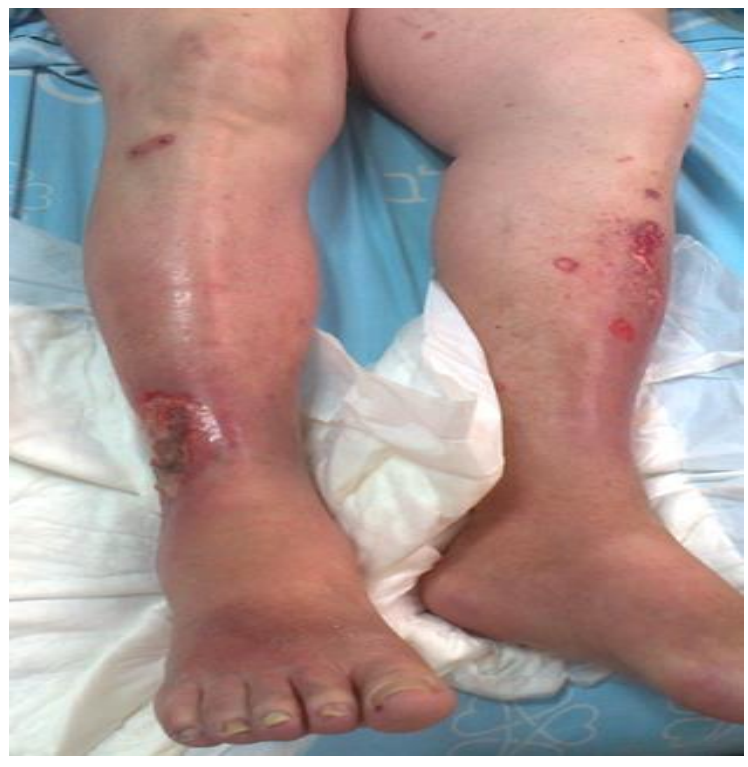

Figure 3: Congestive heart failure, massive leg edema improved on diuretic treatment, stasis dermatitis and bilateral calf ulcers 


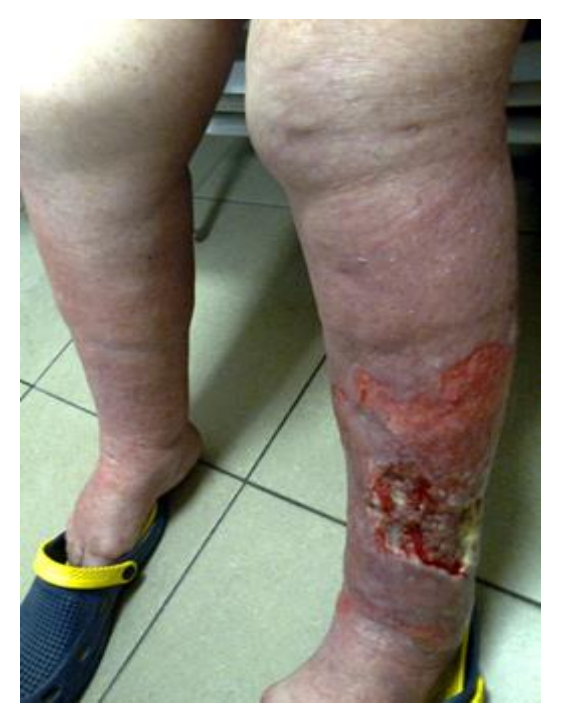

Figure 4: Erythema nodosum migrans

Chronic edema/lymphedema is at the crossroad of the events which initiate stasis dermatitis and stasis ulcers. The subsequent chain of events in the pathophysiology bear also resemblance, indifferent of etiology. To the clinician, the traits of stasis dermatitis irrespective of etiology may be dominant, however, particulars of the primary disorder may suffice to indicate the primary underlying disorder (Table 1 and Figures 1-4). In the management of chronic stasis dermatitis and stasis ulcers, elastic compression and topical ulcer and skin care are similar for either disorder. Still, tailoring treatment to the underlying cause is necessary. Treatment with diuretics is not appropriate for patients with chronic lymphedema because the primary driver of pathophysiology is inflammation and increased interstitial oncotic pressure due to accumulation of macromolecular debris. In patients hospitalized for massive multifactorial edema, there is often combined venous and lymphatic failure. When these patients are treated with bed rest, elevation, manual lymph drainage, and compression, their response to acute treatment can be augmented by temporarily administering a short-course diuretic [21].

Stasis dermatitis and ulcers, of venous or other origin, are usually the consequence of capillary hyperfiltration and the relative insufficiency of the lymphatic system to drain the overload. As recently accepted in consensus, any edema is lymphedema [12], and stasis dermatitis as well as stasis ulcer are the possible consequences. Therefore, a unifying concept comprising the spectrum of etiologies timely to be adopted and instructive to the practitioner.

\section{Conclusions}

Chronic stasis dermatitis, usually confined to the lower legs, is a complication of longstanding interstitial edema and inflammation, due either to venous hypertension or to other disorders, all causing excessive lymph overload. Indifferent to the initial trigger, the pathophysiological chain of subsequent events is similar. The mechanisms which cause stasis dermatitis can also generate skin ulcers superimposed on chronic stasis dermatitis. Such ulcers are called in the appropriate context "venous ulcer" or, in a different context, stasis ulcers anonymous to this date but deserving a suitable name. The distinction between venous and other stasis ulcers is generally possible at the bedside based on characteristics of the ulcers and the clinical context. A venous ultrasound examination may be required to unmask cases of occult venous reflux. Also, general measures of therapy are similar for either disorder, such as elastic compression and topical ulcer and skin care. A unifying concept comprising the spectrum venous and other stasis ulcers appears to be instructing in promoting adequate management.

\section{References}

1. Diaz JA, Henke PK. (2019). Pathophysiology of stasis dermatitis and dermal fibrosis. In: Rutherford's Vascular Surgery and Endovascular Therapy. Elsevier, 2019; 9:97104.e3.

2. James WD, Elston DM, Treat JR, Rosenbach MA, Neuhaus IM. (2020). Cutaneous vascular diseases. In: Andrews' Diseases of the Skin, 13th edition, Elsevier, 2020; 35:813-861.e5.

3. Goldman MP. (2017). Adverse sequelae and complications of venous hypertension. Sclerotherapy, 6th edition, Elsevier, $2017 ; 2: 27-54$

4. Bergan JJ, Schmid-Schönbein GW, Coleridge Smith PD, Nicolaides AN, Boisseau MR, Ekl of B. (2006). N Engl J Med. 2006; 355:488-498.

5. Bergan J. (2007). Molecular mechanisms in chronic venous insufficiency. Ann Vasc Surg. 2007; 21:260-266.

6. Pocock ES, Alsaigh T, Mazor R, Schmid-Schonbein GW. (2014). Cellular and molecular basis of venous insufficiency. Vasc Cell. 2014; 6:24.

7. Sorescu GP, Sykes M, Weiss D, Platt MO, Saha A, Hwang J, Boyd N, Boo YC, Vega JD, Taylor WR, et al. (2003). Bone morphogenic protein 4 produced in endothelial cells by oscillatory shear stress stimulates an inflammatory response. J Biol Chem. 2003; 278:31128-31135.

8. Sundaresan S, Migden MR, Silapunt S. Stasis dermatitis: pathophysiology, evaluation, and management. Am J Clin Dermatol. 2017; 18:383-390.

9. Moffatt CJ, Franks PJ, Doherty DC, Williams AF, Badger C, Jeffs E, Bosanquet N, Mortimer PS. (2003). Lymphedema: An underestimated health problem. Q J Med 2003; 96:731-738.

10. Mortimer PS. (2000). ABC of arterial and venous disease: Swollen lower limb - 2: Lymphedema. Br Med J 2000; 320:1527-1529.

11. Casley-Smith JR. (1988). The pathophysiology of lymphedema and the action of benzo-pyrones in reducing it. Lymphology.1988; 21:190-194.

12. Keast DH, Moffatt C, Janmohammad A. (2019). Lymphedema Impact and Prevalence International Study: The Canadian Data. Lymphat Res Biol. 2019; 17:178-186.

13. Barron GS, Jacob SE, Kirsner RS. (2007). Dermatologic complications of chronic venous disease: medical management and beyond. Ann Vasc Surg 2007; 21:652-662. 
14. Gosnell AL. (2009). Stasis dermatitis as a complication of amlodipine therapy. J Drugs Dermatol 2009; 8:135-137.

15. Naschitz JE. (2021). Alpelisib induced hyperglycemia, rash, and calf ulcers. Personal communication. 2021, October.

16. Son A, O'Donnell Jr TF, Izhakoff J, Gaebler JA, Niecko T, Iafrati MA. (2019). Lymphedema-associated comorbidities and treatment gap. J Vasc Surg Venous Lymphat Disord. 2019; 7:724-730.

17. Hoffman, MD. (2013). Atypical ulcers. Dermatol Ther, 2013; 26:222-235.
18. Papi M, Papi C. (2016). Vasculitic ulcers at the etiology and tailor the therapy accordingly. Int J Low Extrem Wounds, 2016; 5:6-16.

19. Liaw FY, Huang CF, Wu YC, Wu BY. (2012). Elephantiasis nostras verrucosa: swelling with verrucose appearance of lower limbs. Can Fam Physician. 2012; 58:e551-e553.

20. Mokhtari F, Abtahi-Naeini B, Porazizi N. (2014). Erythema nodosum migrans successfully treated with indomethacin: A rare entity. Adv Biomed Res. 2014; 3:264.

21. Bonkemeyer Millan S, Gan R, Townsend PE. (2019). Venous ulcers: diagnosis and treatment. Amer Family Physician, 2019; 100:298-305.
This work is licensed under Creative Commons Attribution 4.0 License

To Submit Your Article Click Here: $\quad$ Submit Manuscript

DOI: $10.31579 / 2690-1919 / 211$
Ready to submit your research? Choose Auctores and benefit from:

$>$ fast, convenient online submission

$>$ rigorous peer review by experienced research in your field

$>$ rapid publication on acceptance

$>$ authors retain copyrights

$>$ unique DOI for all articles

$>$ immediate, unrestricted online access

At Auctores, research is always in progress.

Learn more https://auctoresonline.org/journals/journal-of-clinical-research-andreports 